\title{
Quotient Square Sum Cordial Labeling
}

\author{
T.M. Selvarajan, Swapna Raveendran
}

Abstract--- Let $G=(V, E)$ be a simple graph and $\varphi: V \rightarrow\{1,2$, $\ldots|V|\}$ be a bijection, for each edge uv assigned the label 1 if $\left[\frac{[\varphi(u)]^{2}+[\varphi(v)]^{2}}{(\varphi(u)+\varphi(v))}\right\rfloor$ is odd and 0 if $\left\lfloor\frac{[\varphi(u)]^{2}+[\varphi(v)]^{2}}{(\varphi(u)+\varphi(v))}\right\rfloor$ is even. $\varphi$ is called quotient square sum cordial labeling if $\left|e_{\varphi}(0)-e_{\varphi}(1)\right| \leq$ 1 , where $e_{\varphi}(0)$ and $e_{\varphi}(1)$ denote the number of edges labeled with 0 and labeled with 1 respectively. A graph which admits $a$ quotient square sum cordial labeling is called quotient square sum cordial graph. In this paper path $P_{n}$, cycle $C_{n}$, star $K_{1, n}$, friendship graph $F_{n}$, bistar $B_{n, n}, C_{4} \cup P_{n}, K_{m, 2}$ and $K_{m, 2} \cup P_{n}$ are shown to be quotient square sum cordial labeling .

Keywords--- Quotient Square Sum Cordial Labeling, Friendship Graph, Wheel Graph and Double Fan Graph.

AMS Mathematical Subject Classification (2010)--- $05 C 78$

In this paper, the authors defined quotient square sum cordial labeling and prove the existence or nonexistence of quotient square sum cordial labeling for some families of graphs.

\section{INTRODUCTION}

The field of Graph Theory plays an important role in various areas of pure and applied sciences. A labeling of a graph $\mathrm{G}$ is a mapping that carries a set of graph elements, usually the vertices and edges into a set of numbers, usually integers. Many kinds of labeling have been studied and an excellent survey of graph labeling can be found in [3].

Graph Labeling of a graph $G$ is an assignment of integers either to the vertices or edges or both subject to certain conditions. Graph labeling is a very powerful tool that eventually makes things in different fields very ease to be handled in mathematical way. Nowadays graph labeling has much attention from different brilliant researches in graph theory which has rigorous applications in many disciplines, e.g., communication networks, coding theory, optimal circuits layouts, astronomy, radar and graph decomposition problems. Recently the concept of prime graceful labeling was introduced by T.M. Selvarajan and R. Subramoniam in the year 2018. They studied the prime graceful labeling of various graphs in [5]. In [6] M. Sudha and A. Chandra Babu studied the relation between Different types of Graceful Graphs The symbol [x ] stands for largest integer less than or equal to $\mathrm{x}$.

\section{DISCUSSION \& RESULTS}

\section{Definition 1.1}

Let $\mathrm{G}$ be a $(\mathrm{p}, \mathrm{q})$ graph. Let $\varphi: \mathrm{V}(\mathrm{G}) \rightarrow\{1,2,3, \ldots \mathrm{p}\}$ be a one- one map. For each edge uv assign the label $\left[\frac{\varphi(u)}{\varphi(v)}\right]$ or [ $\left.\frac{\varphi(v)}{\varphi(u)}\right]$ according as $\varphi(\mathrm{u}) \geq \varphi(\mathrm{v})$ or $\varphi(\mathrm{v}) \leq \varphi(\mathrm{u})$. $\mathrm{f}$ is called a quotient cordial labeling of $\mathrm{G}$, if $\left|e_{\varphi}(0)-e_{\varphi}(1)\right| \leq 1$, where $e_{\varphi}(0)$ and $e_{\varphi}(1)$ respectively denote the number of edges

Revised Manuscript Received on July 10, 2019.

T.M. Selvarajan, Department of Mathematics, Noorul Islam Centre for Higher Education, Kanyakumari, T.N, India

Swapna Raveendran, Department of Mathematics, Noorul Islam Centre for Higher Education, Kanyakumari, T.N, India. labeled with even integers and number of edges labeled with odd integers. A graph with a quotient cordial labeling is called quotient cordial graph.

\section{Definition 1.2}

Let $\mathrm{G}=(\mathrm{V}, \mathrm{E})$ be a simple graph and $\varphi: \mathrm{V} \rightarrow\{1,2, \ldots \mid$ $\mathrm{V} \mid\}$ be a bijection, for each edge uv assigned the label 1 if $\left\lfloor\frac{[\varphi(u)]^{2}+[\varphi(v)]^{2}}{(\varphi(u)+\varphi(v))}\right\rfloor$ is odd and 0 if $\left[\frac{[\varphi(u)]^{2}+[\varphi(v)]^{2}}{(\varphi(u)+\varphi(v))}\right\rfloor$ is even. $\varphi$ is called quotient square sum cordial labeling if $\mid e_{\varphi}(0)-$ $e_{\varphi}(1) \mid \leq 1$, where $e_{\varphi}(0)$ and $e_{\varphi}(1)$ denote the number of edges labeled with 0 and labeled with 1 respectively. A graph which admits a quotient square sum cordial labeling is called quotient square sum cordial graph.

Example 1.3

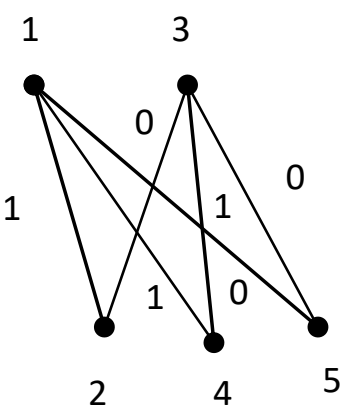

For e(23) (edge 23), $\left\lfloor\frac{(4+9)}{(2+3)}\right\rfloor=\lfloor 2.6\rfloor=2$.

$e_{\varphi}(0)=3$ and $e_{\varphi}(1)=3 \Rightarrow\left|e_{\varphi}(0)-e_{\varphi}(1)\right| \leq 1$, therefore $\mathrm{K}_{2,3}$ admits quotient square sum cordial labeling .

Example 1.4

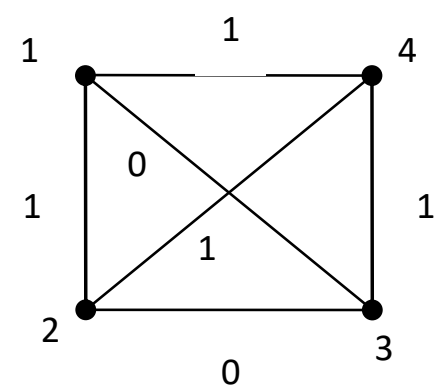

$e_{\varphi}(0)=2$ and $e_{\varphi}(1)=4 \Rightarrow\left|e_{\varphi}(0)-e_{\varphi}(1)\right|>1$

The complete graph $\mathrm{K}_{4}$ does not admit quotient square sum cordial labeling.

\section{Theorem 1.5}

For $\mathrm{n} \geq 3$, the path $\mathrm{P}_{\mathrm{n}}$ admits quotient square sum cordial labeling.

\section{Published By:}

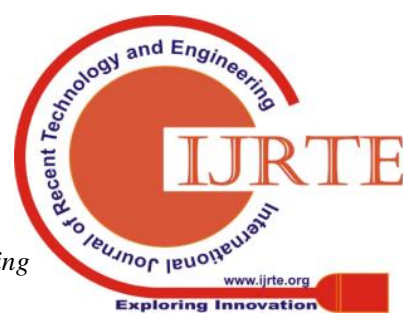




\section{Quotient Square Sum Cordial Labeling}

\section{Proof}

Let $P_{n}=\left\{v_{1}, v_{2}, \ldots, v_{n}\right\}$ be a path with $n \geq 3$, where $v_{k}$ and $\mathrm{v}_{\mathrm{k}+1}$ are adjacent.

Define $:\left\{\mathrm{v}_{1}, \mathrm{v}_{2}, \ldots, \mathrm{v}_{\mathrm{n}}\right\} \rightarrow\{1,2, \ldots, \mathrm{n}\}$ by $\varphi\left(\mathrm{v}_{\mathrm{k}}\right)=\mathrm{k}$. $\frac{\left[\varphi\left(v_{k}\right)\right]^{2}+\left[\varphi\left(v_{k+1}\right)\right]^{2}}{\left[\varphi\left(v_{k}\right)+\varphi\left(v_{k+1}\right)\right]}=\frac{k^{2}+(k+1)^{2}}{\{(k+(k+1)]}=\mathrm{k}+\frac{1}{2}+\frac{1}{4 k+2}$

$\frac{1}{2}+\frac{1}{4 k+2}<1$ for all $\mathrm{k}>0,\left\lfloor\frac{\left[\varphi\left(v_{k}\right)\right]^{2}+\left[\varphi\left(v_{k+1}\right)\right]^{2}}{\left[\varphi\left(v_{k}\right)+\varphi\left(v_{k+1}\right)\right]}\right\rfloor=\mathrm{k}$

$e\left(v_{k} v_{k+1}\right)=\left\{\begin{array}{c}1 \text { if } k \text { is odd } \\ 0 \text { if } k \text { is even }\end{array}\right.$

If $\mathrm{n}$ is even, $\mathrm{e}\left(\mathrm{v}_{1} \mathrm{v}_{2}\right)=1, \mathrm{e}\left(\mathrm{v}_{2} \mathrm{v}_{3}\right)=0, \mathrm{e}\left(\mathrm{v}_{3} \mathrm{v}_{4}\right)=1, \ldots$, $\mathrm{e}\left(\mathrm{v}_{\mathrm{n}-1} \mathrm{v}_{\mathrm{n}}\right)=1$,

then $e_{\varphi}(0)=\frac{n}{2}$ and $e_{\varphi}(1)=\frac{n}{2}-1 \Rightarrow\left|e_{\varphi}(0)-e_{\varphi}(1)\right|=1$

If $\mathrm{n}$ is odd, $\mathrm{e}\left(\mathrm{v}_{1} \mathrm{v}_{2}\right)=1, \mathrm{e}\left(\mathrm{v}_{2} \mathrm{v}_{3}\right)=0, \mathrm{e}\left(\mathrm{v}_{3} \mathrm{v}_{4}\right)=1, \ldots$, and $\mathrm{e}\left(\mathrm{v}_{\mathrm{n}-1} \mathrm{v}_{\mathrm{n}}\right)=0$,

Then $e_{\varphi}(0)=\frac{(n-1)}{2}$ and $e_{\varphi}(1)=\frac{(n-1)}{2} \Rightarrow\left|e_{\varphi}(0)-e_{\varphi}(1)\right|=$ 0 .

\section{Theorem 1.6}

The star graph $\mathrm{K}_{1, \mathrm{n}}$ admits quotient square sum cordial labeling.

\section{Proof}

Let $v_{1}$ be the central vertex of degree $n$.

Let $v_{2}, \ldots, v_{n}, v_{n+1}$ be the $n$ pendant vertices attached to it.

Define $\varphi:\left\{\mathrm{v}_{1}, \mathrm{v}_{2}, \ldots, \mathrm{v}_{\mathrm{n}}, \mathrm{v}_{\mathrm{n}+1}\right\} \rightarrow\{1,2, \ldots, \mathrm{n}, \mathrm{n}+1\}$ by $\varphi\left(\mathrm{v}_{\mathrm{k}}\right)=\mathrm{k}$.

$\frac{\left[\varphi\left(v_{1}\right)\right]^{2}+\left[\varphi\left(v_{k}\right)\right]^{2}}{\left[\varphi\left(v_{1}\right)+\varphi\left(v_{k}\right)\right]}=\frac{1+k^{2}}{[1+k]}=(k-1)+\frac{2}{(k+1)}$

$\frac{2}{(k+1)}<1$ for $\mathrm{k} \geq 2,\left[\frac{\left[\varphi\left(v_{1}\right)\right]^{2}+\left[\varphi\left(v_{k}\right)\right]^{2}}{\left[\varphi\left(v_{1}\right)+\varphi\left(v_{k}\right)\right]}\right\rfloor=k-1$ for $\mathrm{k} \geq 2$

$e\left(v_{1} v_{k}\right)=\left\{\begin{array}{c}1 \text { if } k \text { is even } \\ 0 \text { if } k \text { is odd }\end{array}\right.$

If $\mathrm{n}$ is even, $\mathrm{e}\left(\mathrm{v}_{1} \mathrm{v}_{\mathrm{k}}\right)=1$, for $\mathrm{k}=2,4,6, \ldots, \mathrm{n}$ and $\mathrm{e}\left(\mathrm{v}_{1} \mathrm{v}_{\mathrm{k}}\right)=0$ for $\mathrm{k}=3,5, \ldots,(n+1)$

$e_{\varphi}(0)=\frac{n}{2}$ and $e_{\varphi}(1)=\frac{n}{2} \Rightarrow\left|e_{\varphi}(0)-e_{\varphi}(1)\right|=0$.

If $\mathrm{n}$ is odd, $\mathrm{e}\left(\mathrm{v}_{1} \mathrm{v}_{\mathrm{k}}\right)=1$, for $\mathrm{k}=2,4,6, \ldots,(\mathrm{n}-1)$ and $\mathrm{e}\left(\mathrm{v}_{1} \mathrm{v}_{\mathrm{k}}\right)$ $=0$, for $\mathrm{k}=3,5, \ldots, \mathrm{n}$.

$e_{\varphi}(0)=\frac{(n-1)}{2}$ and $e_{\varphi}(1)=\frac{(n+1)}{2} \Rightarrow\left|e_{\varphi}(0)-e_{\varphi}(1)\right|=1$

\section{Theorem 1.7}

The graph $K_{n, 2}$ is an quotient square sum cordial graph for $\mathrm{n} \neq 3$.

\section{Proof}

The graph $K_{n, 2}$ contains $(n+2)$ vertices and $2 n$ edges.

Label the vertices having degree $n$ of $K_{n, 2}$ with 1 and 2 remaining vertices with integers $3,4,5, \ldots,(\mathrm{n}+2)$.

Define $\varphi:\left\{\mathrm{v}_{1}, \mathrm{v}_{2}, \ldots, \mathrm{v}_{\mathrm{n}}, \mathrm{v}_{\mathrm{n}+1}, \mathrm{v}_{\mathrm{n}+2}\right\} \rightarrow\{1,2, \ldots$,

$\mathrm{n}, \mathrm{n}+1, \mathrm{n}+2\}$ by

$\varphi\left(\mathrm{v}_{1}\right)=1, \varphi\left(\mathrm{v}_{2}\right)=2$ and $\varphi\left(\mathrm{v}_{\mathrm{k}}\right)=\mathrm{k}$ for $3 \leq \mathrm{k} \leq \mathrm{n}+2$.

By Theorem 1.6, $\left[\frac{\left[\varphi\left(v_{1}\right)\right]^{2}+\left[\varphi\left(v_{k}\right)\right]^{2}}{\left[\varphi\left(v_{1}\right)+\varphi\left(v_{k}\right)\right]}\right\rfloor=k-1$ for $\mathrm{k} \geq 2$.

$e\left(v_{1} v_{k}\right)=\left\{\begin{array}{c}1 \text { if } k \text { is even } \\ 0 \text { if } k \text { is odd }\end{array}\right.$

$\frac{\left[\varphi\left(v_{2}\right)\right]^{2}+\left[\varphi\left(v_{k}\right)\right]^{2}}{\left[\varphi\left(v_{2}\right)+\varphi\left(v_{k}\right)\right]}=\frac{4+k^{2}}{[2+k]}=(k-2)+\frac{8}{(k+2)}$

$\frac{8}{(k+2)}<1$ for $\mathrm{k}>6,\left[\frac{\left[\varphi\left(v_{2}\right)\right]^{2}+\left[\varphi\left(v_{k}\right)\right]^{2}}{\left[\varphi\left(v_{2}\right)+\varphi\left(v_{k}\right)\right]}\right\rfloor=k-2$ for $\mathrm{k}>6$.

For $\mathrm{k}>6, e\left(v_{2} v_{k}\right)=\left\{\begin{array}{l}0 \text { if } k \text { is even } \\ 1 \text { if } k \text { is odd }\end{array}\right.$
Case 1 If $\mathrm{n}$ is even $\mathrm{e}\left(\mathrm{v}_{1} \mathrm{v}_{\mathrm{k}}\right)=1$, for $\mathrm{k}=4,6, \ldots, n+2$, $\mathrm{e}\left(\mathrm{v}_{1} \mathrm{v}_{\mathrm{k}}\right)=0$, for $\mathrm{k}=3,5, \ldots,(n-1)$ and $\mathrm{e}\left(\mathrm{v}_{2} \mathrm{v}_{\mathrm{k}}\right)=0$, for $\mathrm{k}$ $=8,10 \ldots, n+2$,

$\mathrm{e}\left(\mathrm{v}_{2} \mathrm{v}_{\mathrm{k}}\right)=1$, for $\mathrm{k}=7,9, \ldots,(n+1)$.

$\mathrm{e}\left(\mathrm{v}_{2} \mathrm{v}_{3}\right)=0, \mathrm{e}\left(\mathrm{v}_{2} \mathrm{v}_{5}\right)=0, \mathrm{e}\left(\mathrm{v}_{2} \mathrm{v}_{4}\right)=1$ and $\mathrm{e}\left(\mathrm{v}_{2} \mathrm{v}_{6}\right)=1$. $e_{\varphi}(0)=\mathrm{n}$ and $e_{\varphi}(1)=\mathrm{n} \Rightarrow\left|e_{\varphi}(0)-e_{\varphi}(1)\right|=0$.

Case 2: If $\mathrm{n}$ is odd and $\mathrm{n} \neq 3$. $\mathrm{e}\left(\mathrm{v}_{1} \mathrm{v}_{\mathrm{k}}\right)=1$, for $\mathrm{k}=4,6, \ldots$, $\mathrm{e}\left(\mathrm{v}_{1} \mathrm{v}_{\mathrm{k}}\right)=0$, for $\mathrm{k}=3,5, \ldots, \mathrm{n}, \mathrm{e}\left(\mathrm{v}_{2} \mathrm{v}_{\mathrm{k}}\right)=0$, for $\mathrm{k}=8,10 \ldots$, $n+2$,

$\mathrm{e}\left(\mathrm{v}_{2} \mathrm{v}_{\mathrm{k}}\right)=1$, for $\mathrm{k}=7,9, \ldots,(n+2)$.

$\mathrm{e}\left(\mathrm{v}_{2} \mathrm{v}_{3}\right)=0, \mathrm{e}\left(\mathrm{v}_{2} \mathrm{v}_{5}\right)=0, \mathrm{e}\left(\mathrm{v}_{2} \mathrm{v}_{4}\right)=1$ and $\mathrm{e}\left(\mathrm{v}_{2} \mathrm{v}_{6}\right)=1$.

$e_{\varphi}(0)=\mathrm{n}$ and $e_{\varphi}(1)=n \Rightarrow\left|e_{\varphi}(0)-e_{\varphi}(1)\right|=0$

\section{Theorem 1.8}

The fan graph $\mathrm{F}_{\mathrm{n}}=\mathrm{Pn}+\mathrm{K}_{1}$ is a quotient square sum cordial graph.

\section{Proof}

The fan graph $\mathrm{F}_{\mathrm{n}}=\mathrm{K}_{1}+\mathrm{P}_{\mathrm{n}}$ contains $n+1$ vertices and $(2 n-1)$ edges. Label the vertex having degree $n$ of $F_{n}$ with 1 and the remaining vertices by $2,3,4, \ldots, n,(n+1)$.

Case 1: If $\mathrm{n}$ is even, using Theorem 1.5 and Theorem 1.6, $e_{\varphi}(0)=\mathrm{n}$ and $e_{\varphi}(1)=(n-1) \Rightarrow\left|e_{\varphi}(0)-e_{\varphi}(1)\right|=1$.

Case 2 If $\mathrm{n}$ is odd, using Theorem 1.5 and Theorem 1.6, $e_{\varphi}(0)=(n-1)$

and $e_{\varphi}(1)=\mathrm{n} \Rightarrow\left|e_{\varphi}(0)-e_{\varphi}(1)\right|=1$. Theorem 1.9. The Double fan graph $\mathrm{F}_{2, \mathrm{n}}$ is a quotient square sum cordial graph for $n \neq 3$.

Proof : The Double fan graph $\mathrm{F}_{2, \mathrm{n}}$ contains $\mathrm{n}+2$ vertices and $(3 n-1)$ edges. Label the vertices having degree $n$ of double fan graph $\mathrm{F}_{2, \mathrm{n}}$ with 1 and 2 , the remaining vertices by $3,4, \ldots, \mathrm{n},(n+1),(n+2)$

Case 1: If $\mathrm{n}$ is even, using Theorem 1.5 and Theorem 1.7, $e_{f}(0)=\frac{3 n}{2}$ and $e_{\varphi}(1)=\frac{3 n}{2}-1 \Rightarrow\left|e_{\varphi}(0)-e_{\varphi}(1)\right|=1$.

Case 2: If $\mathrm{n}$ is odd, using Theorem 1.5 and Theorem 1.7, $e_{\varphi}(0)=\frac{(3 n-1)}{2}$ and $e_{\varphi}(1)=\frac{(3 n-1)}{2} \Rightarrow\left|e_{\varphi}(0)-e_{\varphi}(1)\right|=0$.

\section{Theorem 1.10}

For $\mathrm{n} \geq 3$, the cycle $\mathrm{Cn}$ is a quotient square sum cordial graph.

\section{Proof}

Let $\mathrm{C}_{\mathrm{n}}=\left\{\mathrm{v}_{1}, \mathrm{v}_{2}, \ldots, \mathrm{v}_{\mathrm{n}}, \mathrm{v}_{1}\right\}$ be the cycle, where $\mathrm{v}_{\mathrm{k}}$ and $\mathrm{v}_{\mathrm{k}+1}$ are adjacent,

$\mathrm{v}_{\mathrm{n}}$ and $\mathrm{v}_{1}$ are adjacent for $\mathrm{k}=1,2,3, \ldots, \mathrm{n}$.

case $(\mathbf{i}): \mathrm{n}$ is odd

Define $\varphi:\left\{\mathrm{v}_{1}, \mathrm{v}_{2}, \ldots, \mathrm{v}_{\mathrm{n}}\right\} \rightarrow\{1,2, \ldots, \mathrm{n}\}$ by $\varphi\left(\mathrm{v}_{\mathrm{k}}\right)=\mathrm{k}$ for $\mathrm{k}=1,2,3, \ldots, \mathrm{n}$.

$\frac{\left[\varphi\left(v_{k}\right)\right]^{2}+\left[\varphi\left(v_{k+1}\right)\right]^{2}}{\left[\varphi\left(v_{k}\right)+\varphi\left(v_{k+1}\right)\right]}=\frac{k^{2}+(k+1)^{2}}{\{(k+(k+1)]}=\mathrm{k}+\frac{1}{2}+\frac{1}{4 k+2}$

$\frac{1}{2}+\frac{1}{4 k+2}<1$ for all $\mathrm{k}>0$ 


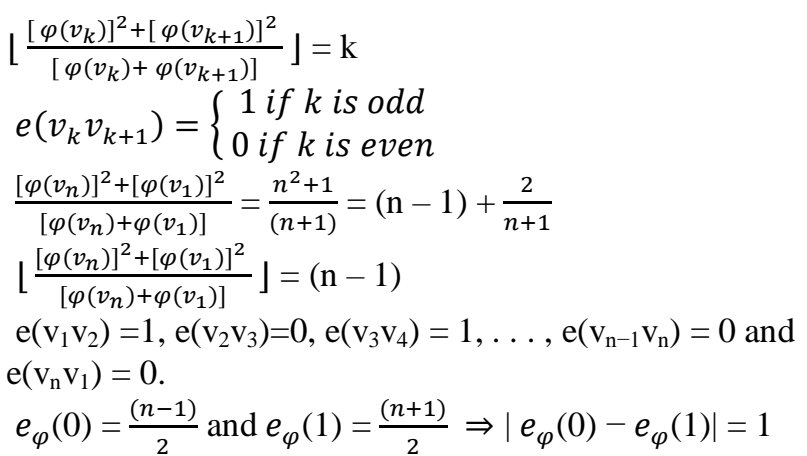

Case (ii) : $n$ is even

Define $\varphi:\left\{\mathrm{v}_{1}, \mathrm{v}_{2}, \ldots, \mathrm{v}_{\mathrm{n}}\right\} \rightarrow\{1,2, \ldots, \mathrm{n}\}$ by

$\varphi\left(\mathrm{v}_{\mathrm{k}}\right)=\left\{\begin{array}{c}k \text { if } 1 \leq k \leq n-4 \\ n \text { if } k=n-3 \\ k-1 \text { if } n-2 \leq k \leq n\end{array}\right.$

$\left[\frac{\left[\varphi\left(v_{k}\right)\right]^{2}+\left[\varphi\left(v_{k+1}\right)\right]^{2}}{\left[\varphi\left(v_{k}\right)+\varphi\left(v_{k+1}\right)\right]}\right\rfloor=\mathrm{k}$ for $1 \leq k \leq n-4$

$\frac{\left[\varphi\left(v_{n-3}\right)\right]^{2}+\left[\varphi\left(v_{n-4}\right)\right]^{2}}{\left[\varphi\left(v_{n-3}\right)+\varphi\left(v_{n-4}\right)\right]}=(\mathrm{n}-2)+\frac{8}{(2 n-4)}$

$\frac{8}{(2 n-4)}<1$ for $\mathrm{n}>6$

$\left[\frac{\left[\varphi\left(v_{n-3}\right)\right]^{2}+\left[\varphi\left(v_{n-4}\right)\right]^{2}}{\left[\varphi\left(v_{n-3}\right)+\varphi\left(v_{n-4}\right)\right]}\right\rfloor=(\mathrm{n}-2)$ for $\mathrm{n}>6$

$\frac{\left[\varphi\left(v_{n-3}\right)\right]^{2}+\left[\varphi\left(v_{n-2}\right)\right]^{2}}{\left[\varphi\left(v_{n-3}\right)+\varphi\left(v_{n-2}\right)\right]}=(n-1)-\frac{1}{2}+\frac{9}{(4 n-3)}$

$-\frac{1}{2}+\frac{9}{(4 n-3)}<1$ for $\mathrm{n}>3$

$\left\lfloor\frac{\left[\varphi\left(v_{n-3}\right)\right]^{2}+\left[\varphi\left(v_{n-2}\right)\right]^{2}}{\left[\varphi\left(v_{n-3}\right)+\varphi\left(v_{n-2}\right)\right]}\right\rfloor=(n-1)$ for $\mathrm{n}>3$

$\frac{\left[\varphi\left(v_{1}\right)\right]^{2}+\left[\varphi\left(v_{n}\right)\right]^{2}}{\left[\varphi\left(v_{1}\right)+\varphi\left(v_{n}\right)\right]}=(n-2)+\frac{2}{n}$

$\left\lfloor\frac{\left[\varphi\left(v_{1}\right)\right]^{2}+\left[\varphi\left(v_{n}\right)\right]^{2}}{\left[\varphi\left(v_{1}\right)+\varphi\left(v_{n}\right)\right]}\right\rfloor=(n-2)$

$\mathrm{e}\left(\mathrm{v}_{1} \mathrm{v}_{2}\right)=1, \mathrm{e}\left(\mathrm{v}_{2} \mathrm{v}_{3}\right)=0, \mathrm{e}\left(\mathrm{v}_{3} \mathrm{v}_{4}\right)=1, \ldots, \mathrm{e}\left(\mathrm{v}_{\mathrm{n}-4} \mathrm{v}_{\mathrm{n}-3}\right)=0$,

$\mathrm{e}\left(\mathrm{v}_{\mathrm{n}-3} \mathrm{v}_{\mathrm{n}-2}\right)=1$,

$\mathrm{e}\left(\mathrm{v}_{\mathrm{n}-1} \mathrm{v}_{\mathrm{n}}\right)=0$ and $\mathrm{e}\left(\mathrm{v}_{\mathrm{n}} \mathrm{v}_{1}\right)=0$.

$e_{\varphi}(0)=\frac{n}{2}$ and $e_{\varphi}(1)=\frac{n}{2} \Rightarrow\left|e_{\varphi}(0)-e_{\varphi}(1)\right|=0$.

\section{Theorem 1.11}

For $\mathrm{n}>3$, the wheel graph $\mathrm{W}_{\mathrm{n}}=\mathrm{K}_{1}+\mathrm{C}_{\mathrm{n}}$ admits quotient square sum cordial labeling.

\section{Proof}

The wheel graph $\mathrm{W}_{\mathrm{n}}=\mathrm{K}_{1}+\mathrm{C}_{\mathrm{n}}$ contains $n+1$ vertices and $2 \mathrm{n}$ edges. Label the vertex having degree $\mathrm{n}$ of $\mathrm{W}_{\mathrm{n}}$ with 1 and the remaining vertices by $2,3,4, \ldots, \mathrm{n},(n+1)$.

\section{Case 1}

If $\mathrm{n}$ is even, using Theorem 1.6 and Theorem 1.11, $e_{\varphi}(0)$ $=\mathrm{n}$

and $e_{\varphi}(1)=n \Rightarrow\left|e_{\varphi}(0)-e_{\varphi}(1)\right|=0$.

Case 2

If $\mathrm{n}$ is odd, using Theorem 1.6 and Theorem 1.11, $e_{\varphi}(0)$ $=n$

$\Rightarrow\left|e_{\varphi}(0)-e_{\varphi}(1)\right|=0$. and $e_{\varphi}(1)=\mathrm{n}$

\section{Example 1.12}
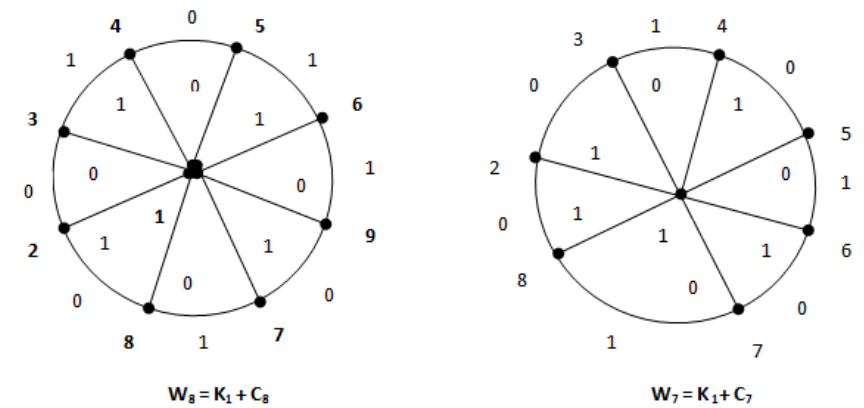

Theorem 1.13

The bistar graph $\mathrm{B}_{\mathrm{n}, \mathrm{n}}$ admits quotient square sum cordial labeling.

\section{Proof}

Bistar graph $B_{n, n}$ contains $(2 n+2)$ vertices and $(2 n+1)$ edges.

Let $|\mathrm{V}(\mathrm{G})|=2 n+2 ;|\mathrm{E}(\mathrm{G})|=2 n+1$. Bistar graph $\mathrm{B}_{\mathrm{n}, \mathrm{n}}$ has exactly two vertices of degree $n$, let them be $u_{0}$ and $v_{0}$.

Define $\varphi:\left\{\mathrm{v}_{0}, \mathrm{v}_{1}, \mathrm{v}_{2}, \ldots, \mathrm{v}_{\mathrm{n}}, \mathrm{u}_{0}, \mathrm{u}_{1}, \mathrm{u}_{2}, \ldots, \mathrm{u}_{\mathrm{n}}\right\} \rightarrow\{1,2, \ldots$ ., $2 \mathrm{n}, 2 n+1,2 n+2\}$ by $\varphi\left(\mathrm{v}_{0}\right)=1, \varphi\left(\mathrm{u}_{0}\right)=2, \varphi\left(\mathrm{v}_{\mathrm{i}}\right)$

$=k+2 ; 1 \leq k \leq n$ and

$\varphi\left(\mathrm{u}_{\mathrm{k}}\right)=n+k ; 3 \leq k \leq(n+2)$

$\frac{\left[\varphi\left(v_{1}\right)\right]^{2}+\left[\varphi\left(v_{k}\right)\right]^{2}}{\left[\varphi\left(v_{1}\right)+\varphi\left(v_{k}\right)\right]}=\frac{1+k^{2}}{[1+k]}=(k-1)+\frac{2}{(k+1)}$

$\frac{2}{(k+1)}<1$ for $\mathrm{k} \geq 2$

$\left[\frac{\left[\varphi\left(v_{1}\right)\right]^{2}+\left[\varphi\left(v_{k}\right)\right]^{2}}{\left[\varphi\left(v_{1}\right)+\varphi\left(v_{k}\right)\right]}\right\rfloor=k-1$ for $\mathrm{k}>2$

$\frac{\left[\varphi\left(u_{0}\right)\right]^{2}+\left[\varphi\left(u_{k}\right)\right]^{2}}{\left[\varphi\left(u_{0}\right)+\varphi\left(u_{k}\right)\right]}=\frac{4+(n+k)^{2}}{[2+n+k]}=(n+k-2)+\frac{8}{(n+k+2)}$

$\frac{8}{(n+k+2)}<1$ for all $\mathrm{k}>3$

$\left[\frac{\left[\varphi\left(u_{0}\right)\right]^{2}+\left[\varphi\left(u_{k}\right)\right]^{2}}{\left[\varphi\left(u_{0}\right)+\varphi\left(u_{k}\right)\right]}\right\rfloor=(n+k-2)$

If $\mathrm{n}$ is even,

$e\left(v_{0} v_{k}\right)=\left\{\begin{array}{c}1 \text { if } k \text { is even } \\ 0 \text { if } k \text { is odd }\end{array}\right.$ and $e\left(u_{0} u_{k}\right)=\left\{\begin{array}{c}0 \text { if } k \text { is even } \\ 1 \text { if } k \text { is odd }\end{array}\right.$ $\mathrm{e}\left(\mathrm{u}_{0} \mathrm{v}_{0}\right)=1, \mathrm{e}\left(\mathrm{v}_{0} \mathrm{v}_{\mathrm{k}}\right)=1$, for $\mathrm{k}=2,4,6, \ldots, \mathrm{n}$ and $\mathrm{e}\left(\mathrm{v}_{1} \mathrm{v}_{\mathrm{k}}\right)=0$, for $\mathrm{k}=1,3,5, \ldots,(n-1)$

$e_{\varphi}(0)=\frac{n-2}{2}$ and $e_{\varphi}(1)=\frac{n}{2} \Rightarrow\left|e_{\varphi}(0)-e_{\varphi}(1)\right|=1$.

If $\mathrm{n}$ is odd

$e\left(v_{0} v_{k}\right)=\left\{\begin{array}{c}1 \text { if } k \text { is even } \\ 0 \text { if } k \text { is odd }\end{array}\right.$ and $e\left(u_{0} u_{k}\right)=\left\{\begin{array}{c}1 \text { if } k \text { is even } \\ 0 \text { if } k \text { is odd }\end{array}\right.$ $\mathrm{e}\left(\mathrm{v}_{1} \mathrm{v}_{\mathrm{k}}\right)=1$, for $\mathrm{k}=2,4,6, \ldots,(n-1)$ and $\mathrm{e}\left(\mathrm{v}_{1} \mathrm{v}_{\mathrm{k}}\right)=0$, for $\mathrm{k}$ $=3,5, \ldots, \mathrm{n}$.

$e_{\varphi}(0)=\frac{(n-1)}{2}$ and $e_{\varphi}(1)=\frac{(n-1)}{2} \Rightarrow\left|e_{\varphi}(0)-e_{\varphi}(1)\right|=0$

\section{Theorem 1.14}

The friendship graph $F_{n}^{(3)}$ admits quotient square sum cordial labeling.

\section{Proof}

The friendship graph $F_{n}^{(3)}$, is a planar undirected graph with $2 n+1$ vertices and $3 n$ edges constructed by joining $n$ copies of the triangle graph $\mathrm{T}$ with a common vertex.

\section{Published By:}




\section{Quotient Square Sum Cordial Labeling}

Let $v_{1}$ be the central vertex of degree $2 n$.

Friendship graph contains $n$ triangles $T_{1}, T_{2}, \ldots, T_{n}$.

Define $f: V(G) \rightarrow\{1,2, \ldots, 2 n, 2 n+1\}$ by $\varphi\left(v_{1}\right)=1$, label the outer

vertices of the triangles $\mathrm{T}_{1}, \mathrm{~T}_{3}, \mathrm{~T}_{5}, \ldots$ by $\varphi\left(v_{k}\right)=2 k, 1 \leq$ $k \leq n$ and label the outer vertices of the triangles $\mathrm{T}_{2}, \mathrm{~T}_{4}, \mathrm{~T}_{6}$, $\ldots$ by $\varphi\left(v_{k}\right)=2 k+1,1 \leq k \leq n$.

$\frac{\left[\varphi\left(v_{1}\right)\right]^{2}+\left[\varphi\left(v_{k}\right)\right]^{2}}{\left[\varphi\left(v_{1}\right)+\varphi\left(v_{k}\right)\right]}=\frac{1+k^{2}}{[1+k]}=(k-1)+\frac{2}{(\square+l)}$

$\frac{2}{(k+1)}<1$ for $\mathrm{k} \geq 2$

$\left[\frac{\left[\varphi\left(v_{1}\right)\right]^{2}+\left[\varphi\left(v_{k}\right)\right]^{2}}{\left[\varphi\left(v_{1}\right)+\varphi\left(v_{k}\right)\right]}\right\rfloor=(k-1)$ for $\mathrm{k} \geq 2$

$\frac{\left[\varphi\left(v_{k}\right)\right]^{2}+\left[\varphi\left(v_{k+1}\right)\right]^{2}}{\left[\varphi\left(v_{k}\right)+\varphi\left(v_{k+1}\right)\right]}=\frac{k^{2}+(k+2)^{2}}{\{(k+(k+2)]}=(k+1)+\frac{1}{k+1}$

$\frac{1}{k+1}<1$ for all $\mathrm{k}>1$

$\left\lfloor\frac{\left[\varphi\left(v_{k}\right)\right]^{2}+\left[\varphi\left(v_{k+1}\right)\right]^{2}}{\left[\varphi\left(v_{k}\right)+\varphi\left(v_{k+1}\right)\right]}\right\rfloor=(k+1)$

Case 1

If $\mathrm{n}$ is even, $e_{\varphi}(0)=\frac{3 n}{2}$ and $e_{\varphi}(1)=\frac{3 n}{2} \Rightarrow \mid e_{\varphi}(0)-$ $e_{\varphi}(1) \mid=0$.

Case 2

If $\mathrm{n}$ is odd, $e_{\varphi}(0)=\frac{3 n-1}{2}$ and $e_{\varphi}(1)=\frac{3 n+1}{2}$

$\Rightarrow\left|e_{\varphi}(0)-e_{\varphi}(1)\right|=1$.

\section{Theorem 1.15}

The friendship graph $F r_{n}^{(3)}$ admits quotient square sum cordial

labeling .

\section{Proof}

The friendship graph $\mathrm{Fr}_{n}^{(3)}$ is an undirected graph with $3 n+1$ vertices and $5 n$ edges constructed by joining $n$ copies of the fan graph $\mathrm{F}_{3}$ with a common vertex.

Let $v_{1}$ be the central vertex of degree $3 n$.

Define $\varphi: \mathrm{V}(\mathrm{G}) \rightarrow\{1,2, \ldots, 2 \mathrm{n}, \ldots, 3 \mathrm{n}, 3 n+1\}$ by $\varphi\left(\mathrm{v}_{1}\right)=1$, label the outer vertices $v_{2}, v_{3}, \ldots, v_{3 n}, v_{3 n+1}$ of the friendship graph $\mathrm{Fr}_{n}^{(3)}$

by $\varphi\left(v_{k}\right)=k, 2 \leq k \leq 3 n+1$

$\left[\frac{\left[\varphi\left(v_{1}\right)\right]^{2}+\left[\varphi\left(v_{k}\right)\right]^{2}}{\left.\varphi\left(v_{1}\right)+\varphi\left(v_{k}\right)\right]}\right\rfloor=(k-1)$ for $\mathrm{k} \geq 2$

$\frac{\left[\varphi\left(v_{k}\right)\right]^{2}+\left[\varphi\left(v_{k+1}\right)\right]^{2}}{\left[\varphi\left(v_{k}\right)+\varphi\left(v_{k+1}\right)\right]}=\mathrm{k}+\frac{1}{2}+\frac{1}{4 k+2},\left[\frac{\left[\varphi\left(v_{k}\right)\right]^{2}+\left[\varphi\left(v_{k+1}\right)\right]^{2}}{\left[\varphi\left(v_{k}\right)+\varphi\left(v_{k+1}\right)\right]}\right]=\mathrm{k}$

Case 1:If $\mathrm{n}$ is even , $e_{\varphi}(0)=\frac{5 n}{2}$ and $e_{\varphi}(1)=\frac{5 n}{2} \Rightarrow \mid e_{\varphi}(0)$ $-e_{\varphi}(1) \mid=0$

Case 2: If $\mathrm{n}$ is odd, $e_{\varphi}(0)=\frac{5 n-1}{2}$ and $e_{\varphi}(1)=\frac{5 n+1}{2}$

$\Rightarrow\left|e_{\varphi}(0)-e_{\varphi}(1)\right|=1$.

\section{Example 1.16}

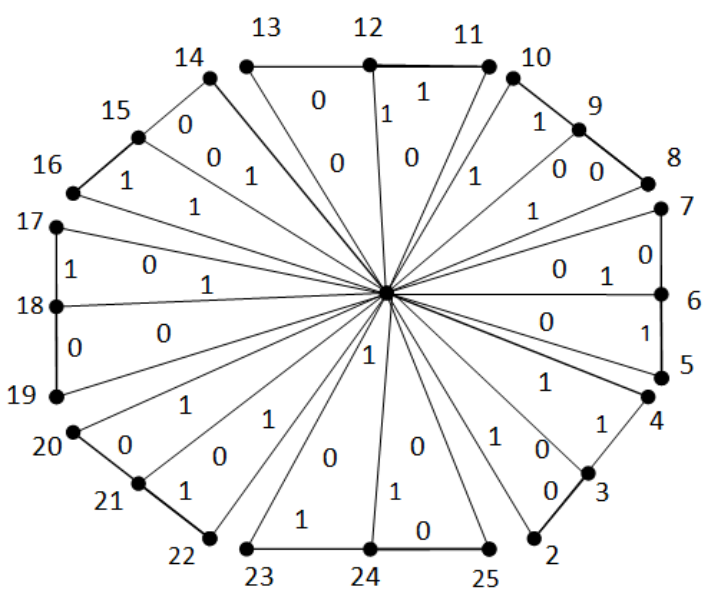

The friendship graph $\mathrm{Fr}_{8}^{(3)}$ $e_{\varphi}(0)=20$ and $e_{\varphi}(1)=20 \Rightarrow\left|e_{\varphi}(0)-e_{\varphi}(1)\right|=0$.

\section{Theorem 1.17}

The graph $\mathrm{P}_{\mathrm{n}} \cup \mathrm{K}_{\mathrm{m}, 2}(\mathrm{~m} \neq 3)$ is a quotient square sum cordial graph.

Proof

The graph $\mathrm{P}_{\mathrm{n}} \cup \mathrm{K}_{\mathrm{m}, 2}$ contains $(n+m+2)$ vertices and $(2 m+n-1)$ edges .Using Theorem 1.5 and Theorem 1.7, $\mathrm{P}_{\mathrm{n}} \cup \mathrm{K}_{\mathrm{m}, 2}$ admits quotient square sum cordial labeling.

Theorem 1.18

The graph $\mathrm{P}_{\mathrm{n}} \cup \mathrm{C}_{4}$ is a quotient square sum cordial graph.

Proof

The graph $\mathrm{P}_{\mathrm{n}} \cup \mathrm{C}_{4}$ contains $(n+4)$ vertices and $(n+3)$ edges .

Label the vertices of $\mathrm{C}_{4}$ by $1,3,2,4$ and the remaining vertices

by $5,6, \ldots,(n+4)$.

For $\mathrm{C}_{4}, e_{\varphi}(0)=e_{\varphi}(1)=2$

Using Theorem 1.5,

$\mathrm{P}_{\mathrm{n}} \cup \mathrm{C}_{4}$ admits quotient square sum cordial labeling.

\section{CONCLUSION}

The investigation of labeled graph is very important due to its various applications in many fields. It is very interesting to study the various types of graphs which admits quotient square sum cordial labeling. It is an open area of research to discuss some more similar results for various graphs.

\section{REFERENCES}

1. F. Harary, Graph Theory, Addison-Wesley, Reading, Mass, (1969).

2. J.A.Bondy and U.S.R.Murthy, Graph Theory and Applications, (North-Holland), Newyork (1976).

3. J.A.Gallian, A Dynamic Survey of Graph Labeling, The Electronic Journal of Combinatorics, 18th edition, Dec 7, 2015. 
5. Rosa A, On Certain Valuations of the vertexs of a Graph, Theory of Graphs (Internat. Symposium, Rome, July 1966), Gordon and Breach, N.Y and Dunod Paris, 394355.

6. T.M. Selvarajan and R. Subramoniam. Prime Graceful Labeling. International Journal of Engineering \& Technology, [S.1.], v. 7, n. 4.36, p. 750-752, dec. 2018. ISSN 2227-524X

7. M. Sudha and A. Chandra Babu, Relation between Different types of Graceful Graphs, International Journal of Applied Engineering Research, ISSN 0973-4562 Vol. 9 No.26 (2014) pp. 9331-9333

8. S K Vaidya and N H Shah, Graceful and odd graceful labeling of some graphs. International Journal of Mathematics and Soft Computing, Vol.3, No.1(2013), 61- 68 . 